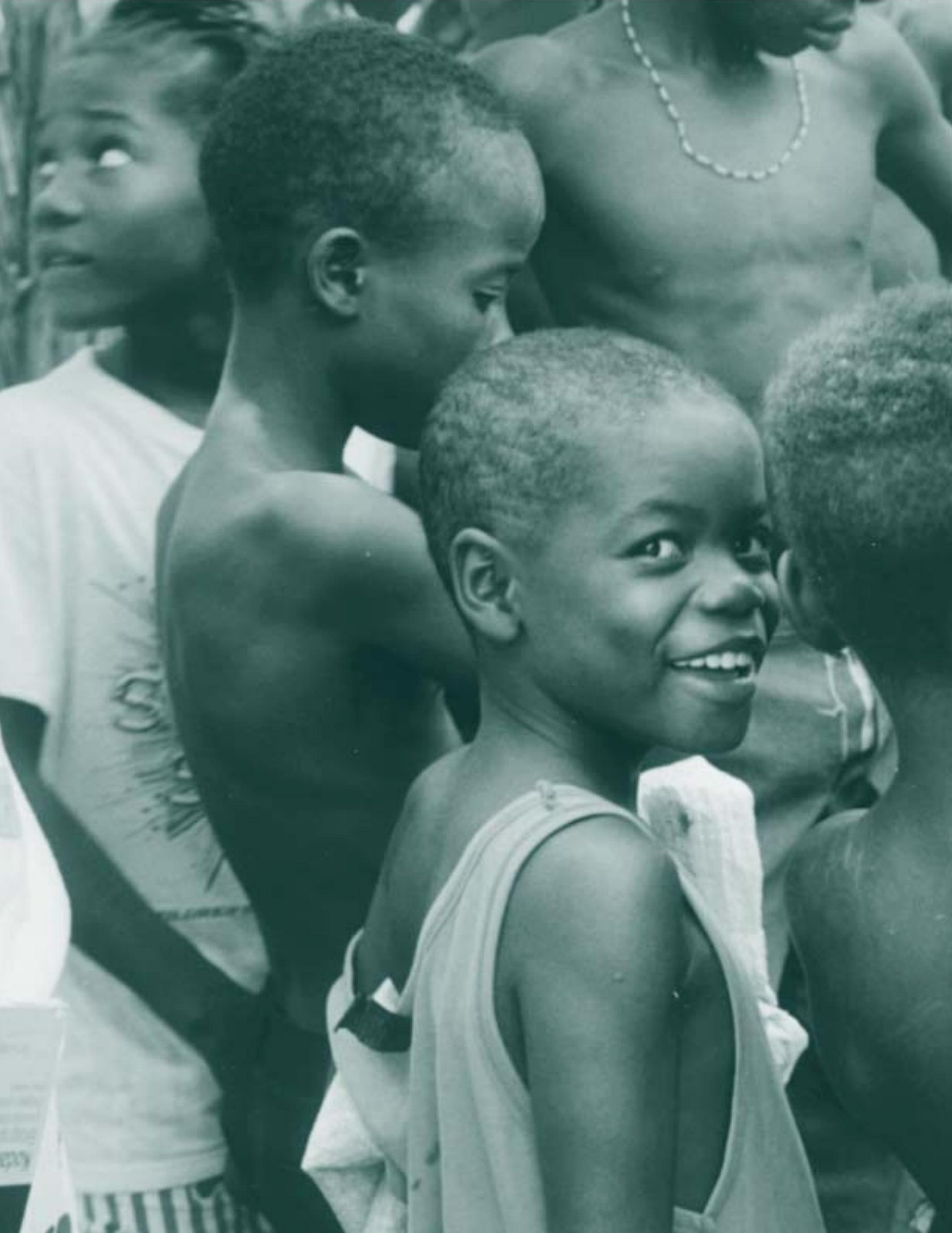




\section{4 proceso de registro vitales en Honduras}

Olvis Adalid Castro, lorena Rivera

MaritZa Andino

\section{INTRODUCCIÓN}

Las Estadísticas Vitales, son el producto registro de los hechos más importantes relacionados con el inicio y el final de la vida de las personas y otros cambios que se puedan producir en el transcurso del tiempo. Estos hechos son: $\mathrm{N}$ acimientos, defunciones, matrimoniosy divorcios.

La importancia de estas estadísticas es la de mantener la información sobre la dinámica poblacional, permitiéndonos conocer el crecimiento y decrecimiento de la población basándose en las tasas de natalidad y mortalidad.

La Estadísticas V itales y los censos de población ayudan al análisis de la estructura y composición de la población y sirven como base para la toma de decisiones y en la formulación de políticas y programas en ben eficio de la población, en los campos de educación (demanda, de centros escolares, equipo, mobiliario, etc.), salud (centros de salud, hospitales, equipo médico, etc.) y programas de vivienda que cumplan con las condiciones para ser habitados.

A demás, los registros vitales sirven como fuente de información a instituciones no gubernamentales e internacionales, que se interesan en conocer la dinámica poblacional para establecer programas de apoyo en beneficio de la población.

En este trabajo se da una mirada a los antecedentes del Sistema de Registros Vitales en $\mathrm{H}$ onduras, pero sobre todo su situación actual, perspectivas, incluyen do al gunas conclusiones y recomendaciones. Sin embargo, es de hacer notar que de los registros vitales el énfasis está en el de los nacimientos y defunciones.

\section{Antecedentes históricos de los registro vitales en H ondu- ras}

Durante el período colonial, la I glesia C atólica, cumplía un papel fundamental al mantener un registro eclesiástico minucioso sobre los eventos vitales, que hoy día representan una gran fuente de información, para poder estimar el comportamiento de algunas variables demográficas en el pasado.

La iglesia realizaba labores de recopilación de información de las comarcas donde tenía jurisdicción, registraba en sus libros los nacimientos y los matrimonios de sus feligreses.

Fue en 1823 que se emitió la primera ley de estadística en $\mathrm{H}$ onduras, bajo el poder Ejecutivo de las Provincias U nidas de Centro
A mérica, en ella, se establecía que sería la Secretaría de Estado la recolectora de la información sobre población, por edad, sexo y oficios, en general con propósitos fiscales, religiosos, militares y administrativos, antes que demográficos (DGEC, 1977).

Posteriormente en los años 1837, 1843 y 1859 se emitieron nuevos decretos y reglamentaciones para ejecutar censos de población y desarrollar registros estadísticos (nacimientos, muertes, matrimonios, migración, producción, costos, etc.), los cuales funcionaron irregularmente a pesar que en cada gobierno departamental, se había creado una Junta de Estadística.

En lo referente a Registros vitales, se dispone de información desde el año 1852 hasta 1881, en forma no continua, estos fueron publicados por año económico en las memorias de G obernación y Justicia (Vallejo A.H. 1889).

El código civil de 1880 reglamenta lo concerniente a las estadísticas vital es (nacimientos, defunciones y matrimonios) y ese mismo año se crea el departamento de Estadística.

En 1881 se levantó el primer censo de población (los anteriores pueden ser considerados como recuentos), este primero, marcó el inicio de uno de los más amplios panoramas censales en A mérica Latina. H abiéndose levantado hasta el 2001, 19 censos.

En el año 1906, fue reformado el código civil de 1880 y se mantuvo vigente hasta 1982, año en que fue emitida la Ley del Registro $\mathrm{N}$ acional de las Personas y que crea la nueva institución del registro Civil y sus R eglamentos.

A diferencia del código de 1880, el de 1906 incluye: Legitimaciones, emancipaciones, separación de cuerpos, divorcios, anulación de matrimonios y declaración de ausencia y discernimiento de guarda y perfecciona el Registro de nacimientos, defunciones, matrimonios y reconocimientos de hijos naturales.

Los registros vitales generados por el Registro $\mathrm{N}$ acional de las Personas y anteriormente por la I glesia C atólica, se han documentado en los Registros A dministrativos del país.

\section{Proceso de generación de estadísticas vitales}

U n sistema de Estadísticas Vitales, se define como, el proceso total de:

a) Recopilación de información por registro, enumeración 0 estimación indirecta relativa a la frecuencia con que ocurren ciertos procesos vitales, así como las características pertinentes a los sucesos 
en sí y de la persona o personas a que concierne. $Y$

b) A nálisis, valoración, presentación y difusión de estos datos en forma estadística.

( $M$ anual de Sistema y M étodo de Estadísticas Vitales, N aciones U nidas, 1985)

Las principales fuentes de datos utilizadas para la recopilación de la información son los censos de población, las encuestas de hogares y los registros administrativos.

En H onduras, el Registro $\mathrm{N}$ acional de las Personas, fue creado según decreto N o. 150 del 17 de noviembre de 1982. Es un organismo del estado con asiento en la Capital de la República y jurisdicción en el territorio nacional, dependiente del Tribunal $\mathrm{N}$ acional de Elecciones.

La oficina central del Registro $\mathrm{N}$ acional de las Personas está organizada de la forma siguiente:
a) Registro Civil
b) Registro Electoral
c) Departamentos
d) Secciones
e) U nidades Internas
f) O ficinas R egionales
g) O ficinas M unicipales y
h) A uxiliares
El R egistro C ivil, es el que registra los hechos y actos del estado civil de las personas desde su nacimiento hasta el fallecimiento. Registra la población del país.

A ctualmente en el país existen:

- 2980 ficinas municipales del Registro Civil.

- 10 O ficinas auxiliares del Registro Civil.

- 27 O ficinas de R egistros C iviles R egionales

La cobertura en las inscripciones de nacimientos es de $90 \%$ (Diario El Heraldo, 11, agosto, 2002).

Los R egistros A dministrativos generan información de Estadísticas Vitales como: N acimientos, defunciones, adopciones, naturalizaciones e inscripciones de matrimonios.

\section{NACIMIENTOS}

Concepto Inscripción: "Es el acto mediante el cual se procede a inscribir lo hechos sujetos a inscripción en el Registro Civil".

Concepto de nacimiento: "Esla expulsión del ser humano que vive desprendido del vientre materno que después de dicha separación, respira o da muestra de vida, constituyéndose en el hecho natural sujeto a inscripción en el Registro Civil".

T érmino L egal: "Los nacimientos ocurridos en el país deberán inscribirse en el registro Civil dentro de los 30 días siguientes al nacimiento".

También se inscribirán dentro del mismo término y ante los agentes diplomáticos y en su efecto los funcionarios consulares, los nacimientos de hijos de padres o madre hondureños, por nacimiento, ocurridos en el extranjero.
Estos últimos además, podrán inscribirse en el Registro Civil $\mathrm{N}$ acional sino lo hubiesen hecho en el extranjero a petición de la parte interesada. Si no declarasen dentro del término antes mencionado, podrá inscribirse siempre no exceda de 5 años presentando las pruebas pertinentes. Están obligados a declarar nacimientos:

- Padre, madres.

- Pariente.

- Personas que hubiesen asistido al parto.

- A Icaldes auxiliares.

- El Juzgado de la N iñez y de la adolescencia, cuando se declara al menos en estado de abandono y no hubiera sido inscrito por sus padres.

Tipos de Inscripción de nacimiento:

a) N acimiento normal o dentro de término legal.

b) $\mathrm{N}$ acimiento de reposición por omisión.

c) N acimiento de reposición de oficio y reinscripción.

Requisitos previos a la inscripción de nacimiento normal:

CaSO 1 NA CIMIENTO NORMAL OCURRIDO EN HOSPITAL

- Reporte de hospital.

- Tarjeta de identidad de los padres.

- Tarjeta de identidad de la persona que comparece (cuando no comparecen los padres).

- Dos testigos con sus tarjetas de identidad.

- C ertificación de partida de nacimiento cuando los padres son menores de edad.

Caso 2 N A CIM IENTO NORM A L OCURRIDO EN CASA

- $M$ ismos que el anterior, excepto que se requiere el reporte de la partera que asistió el parto.

\section{INSCRIPCIÓN DE HIJOS DE PADRES HONDUREÑOS} NACIDOS EN EL EXTRANJERO

Si fue inscrito ante un agente diplomático, funcionario consular de $\mathrm{H}$ onduras en el extranjero, presentará solicitud ante la Dirección de Registro $\mathrm{N}$ acional de las Personas acompañada de la certificación de la Inscripción de nacimiento extendida por el funcionario consular o agente diplomático en el país donde nació. Esta deberá estar debidamente autenticada por la Secretaría de relaciones Exteriores y Gobernación y Justicia. A demás la certificación de la partida de nacimiento de los padres para acreditar la nacionalidad.

Procedimiento: C umplidoslos requisitos, se procede a realizar la inscripción en el libro de nacimientos, de acuerdo a los documentos presentados y a la declaración del compareciente conforme a la información que requiere el contenido de la inscripción. 


\section{DEFUNCIONES:}

Concepto: "Es la ausencia permanente de todas las señales de vida en cualquier momento después de haber tenido lugar del nacimiento vivo".

Este es un hecho de inscripción en el Registro Civil del municipio donde murió.

Término Legal: "Para inscribir una defunción es dentro de los ocho días de haber conocido el hecho".

En los casos en que la defunción ocurra en hospitales, hospicios, asilos, centros educativos, hoteles, cárceles y otros establecimientos análogos, están obligados losj efesy/o admini stradores a transmitir el aviso de las defunciones que ocurran en dichos establecimientos al R egistrador $\mathrm{C}$ ivil, dentro de las veinticuatro horas de haber acaecido. (M anual del Registro Civil e Identificación, 2002)

Los que están obligados a dar parte de la defunción son:

- El cónyuge, Compañero de hogar sobreviviente.

- Los ascendientes y descen dientes mayores de edad.

- Parientes cercanos que vivieron en la casa del difunto.

- M édico que asistió a la persona de cuya defunción se trata.

- El cabeza de familia extraña, en cuya casa ocurrió la muerte y

- La autoridad civil o militar de la jurisdicción cuando la defunción ocurre en despoblado.

Son requisitos para inscribir una defunción:

1) IN SCRIPCIÓN DE FA LLECIDO EN HOSPITAL

a) Reporte de defunción.

b) Tarjeta de identidad del fallecido.

c) Tarjeta de identidad del compareciente.

d) Tarjeta de identidad de dos testigos que den fe de la inscripción y si el inscrito es menor de edad, certificación del acta de nacimiento.

2) IN SCRIPCIÓN DE FA LLECIDO EN CASA

a) Tarjeta de identidad del fallecido y si es menor de edad certificación de acta de nacimiento.

b) Tarjeta de identidad del compareciente y dos testigos con las tarjetas de identidad a quienes les conste el hecho, en caso que no se presente tarjeta de identidad del fallecido.

3) DEFUNCIÓN DE PERSONA FA LLECIDA EN VÍA PÚBLICA

a) Reporte del médico forense 0 autoridad competente, firmado y sellado.

b) Tarjeta de identidad del fallecido; si es menor de edad certificación de acta de nacimiento de éste.

c) Tarjeta de identidad de dostestigos, que den fe de su inscripción.

\section{4) HONDUREÑ O FA LLECIDO EN EL EXTRA NJERO}

Para inscribir una defunción de un hondureño ocurrida en el extranjero, el interesado deberá presentar solicitud ante la Dirección $\mathrm{G}$ eneral del Registro $\mathrm{N}$ acional de las Personas presentando la siguiente documentación: a) Certificación de defunción, extendida por el Registrador Civil del país don de ocurrió la defun ción la cual deberá estar debidamente autenticada por la autoridad consular hondureña, en el exterior, procediendo a su inscripción mediante

b) Resolución de la Dirección General del Registro Nacional de las Personas.

c) Certificación extendida por la autoridad consular debidamente autenticada por los M inisterios de Relaciones Exteriores y G obernación, se procederá a su inscripción mediante oficio por el Departamento de A sesoría Legal.

Cumplidos los requisitos, se procede a registrar la defunción en el libro de defunción de acuerdo a los documentos presentados, y declaración de compareciente conforme a la información de la inscripción.

INSTRUMENTOS DONDE SE REGISTRAN LOS HECHOS VITALES

- Libro de Inscripción de nacimiento.

- Boleta de Inscripción de nacimiento.

- Certificación de acta de nacimiento.

- Libro de inscripción de defunción.

- Constancia de Inscripción de defunción.

- Certificación de acta de defunción.

3. Situación actual del funcionamiento del proceso nacional de estadísticas vitales

A ctualmente, el Registro $\mathrm{N}$ acional de las Personas, como ente dependiente del Tribunal de Elecciones y del que tanto el Director como Sub Director están a cargo del Tribunal de Elecciones, sufre de algunas limitaciones y problemas en su funcionamiento debido al hecho que está ligado al Tribunal nacional de Elecciones, se convierte en un ente propicio a la politización. Esto significa, una baja confiabilidad ya que a pesar de ser la unidad recolectora de información sobre hechos vitales, este registro se ha caracterizado por altos niveles de omisión y errores en los datos recolectados, los que, no satisfacen las necesidades de información, ni reflejan la situación real del país.

Sin embargo, habiéndose creado el Instituto $\mathrm{N}$ acional de Estadísticas y Censos (INE), mediante Decreto N 0. 86-200, y siendo designado como rector de la Estadística $\mathrm{N}$ acional, es el ente encargado de captar estos registros vitales y a pesar de que anteriormente los encargados de los R egistros Civiles no daban mucha importancia a su remisión, se efectúan actualmente negociaciones para lograr obtener copia de la base de datos del R egistro $\mathrm{N}$ acional de las Personas, pero, no está completa por lo que debe continuarse el proceso de digitación y lograr la actualización y calidad de la información a fin de estructurar un Sistema de Información que pueda ser de utilidad parar las autoridades gubernamentales, y otros que tengan interés en dicha información. 
Entre otras, sobresalen las siguientes:

- Falta de interés de la población por efectuar las inscripciones oportunamente.

- Incumplimiento de las autoridades en la aplicación de las sanciones por la no- inscripción.

- $\quad$ Falta de Presupuesto para que el Registrador Civil pueda realizar recorridos periódicos en su jurisdicción, que le permitan recoger información faltante y así evitar las omisiones.

- Casillas en blanco que son dejadas por los registradores y que son Ilenadas posteriormente y no cuando el ciudadano está presente para reportar el hecho vital.

- Inadecuada forma de inscribir en las defunciones la causa de muerte, la cual se atribuye por ejemplo a: tos, machete, dolor de cabeza, pistola, que nos impide una codificación uniforme de acuerdo a las normas internacionales y además determinar las causas real es por las que están muriendo los hondureños.

- $\quad$ Solamente se conocen las causas de muerte publicadas por las encuestas epidemiológicas y registro del M inisterio de Salud.

- O misiones por expediente, que las A Icaldías M unicipales no envían a la oficina del R egistro $\mathrm{N}$ acional de las Personas (matrimonios, divorcios).

- Falta de divulgación de la información, por el alto grado de ingerencia del Tribunal $\mathrm{N}$ acional Electoral, quien considera propiedad de los partidos políticos, la información vital.

- La inadecuada forma de contratación de los R egistradores Civiles, la cual se hace por recomendación política. De hecho los aspirantes a registradores que se presentan a la capacitación en el Registro $\mathrm{N}$ acional de las Personas por 15 días consecutivos son propuestos por los partidos políticos, financiándose personalmente cada uno sus gastos. La capacitación se lleva a cabo cada mes y la reciben grupos que van desde 80 a 200 aspirantes.

- Inexistente coordinación interinstitucional entre el RN P y el M inisterio de Salud que permitan controlar y validar la información vital.

\section{Algunas conclusiones y perspectivas}

A pesar de las dificultades por las que han atravesado el proceso de los R egistros $V$ itales en $\mathrm{H}$ onduras, se observa, un avance significativo en el proceso de generación y continuo mantenimiento de la información, sin embargo, no se cuenta con un Sistema $\mathrm{N}$ acional de Información Vital.

Los obstáculos presentados en el proceso, se han debido en su mayoría tanto a factores económicos culturales como políticos.

La planificación y toma de decisiones gubernamental, debería depender de Estadísticas $V$ itales reales y no de proyecciones, pues la primera reflejan más acertadamente la realidad del país.

Por otra parte es importante decir que al gunas de las expectativas son positivas, ya que existen intentos de organismos internacionales tales como las $\mathrm{N}$ aciones $\mathrm{U}$ nidas, que han realizado estudios para la elaboración de manual es de sistemas y métodos de estadísticas vitales.

Los principios y recomendaciones para un Sistema de Estadísticas $V$ itales de las $\mathrm{N}$ aciones $\mathrm{U}$ nidas fueron aprobados por la C omisión de Estadística en su XVI período de sesiones celebradas en 1970 y después fueron publicados por las $\mathrm{N}$ aciones $U$ nidas.

Existen otros O rganismos internacionales preocupados por la inexistencia de Estadísticas Vitales confiables y actualmente, efectúan esfuerzos vía apoyo financiero y técnico para el mejoramiento del Sistema.

A iniciativa del poder Ejecutivo se tiene en discusión la modificación de la Ley del Registro $\mathrm{N}$ acional de las Personas en el sentido que sea separada y funcione independientemente del Tribunal $\mathrm{N}$ acional de Elecciones, con lo cual se le eliminará el manipuleo político que limita la naturaleza de sus funciones.

La contribución del avance tecnológico un mejoramiento en la calidad de la información y la difusión de la misma.

\section{APARTADO}

\section{PROCESAMIENTO DE LOS HECHOS VITALES}

A I captar la información de los ciudadanos se acude a registrar loshechos vitales, en 37 de los registros municipales hay sistematización y se capta directamente del libro de copiado y en las restantes oficinas al terminarse. El libro que comprende (98) folios, son despren di das las pági nas de copia y son enviadas empastadas a la oficina de Tegucigalpa en donde son digitadas para ingresar al sistema.

El problema existente del R egistro $\mathrm{N}$ acional de las Personas es en la digitación de los libros físicos al sistema, lo cual Ileva un acumulado de varios años, motivo por el cual no se ha conformado el Sistema de Registros Vitales.

El día viernes 21 de noviembre del año 2002, el Instituto $\mathrm{N}$ acional de Estadística firmó un convenio de cooperación institucional con el propósito de apoyar al RN P para organizar el sistema de estadísticas vitales, apoyo que consistirá en asistencia técnica y si fuera el caso apoyo en cuanto a equipo y personal para actualizar la digitación y poder conformar el sistema de información de estadísticas vitales. Cabe agregar que la organización y estructura del RN P ha sido sometida a cambios radicales al haberse aprobado la modificación de la Ley del RN P en el sentido que pasa a ser un ente autónomo, independiente de toda actividad política-partidista.

Según el artículo 54 (reformado) de la Constitución de la República se establece que "El Registro $\mathrm{N}$ acional de las Personas es una institución autónoma, con personalidad jurídica, técnica e independiente, tiene su asiento en la capital de la República y autoridad en el territorio nacional".

Estará administrado por un director y dos subdirectores que serán el ectos por un período de cinco años, por el voto afirmativo de los dos tercios de los votos de los Diputados al Congreso $\mathrm{N}$ acional. Deberán poseer título universitario, las más altas calificaciones técnicas y morales y estarán sujetos a los mismos requisitos e 
inhabilidades que establece la C onstitución de la República para ser M agistrados del Tribunal Superior Electoral".

(Tiempo, Editorial. 2002). La reforma entrará en vigencia a partir de mediados del 2003.

Se adjunta el diagrama de procesos ( Propuesta para reorganización del área de transcripción y control de calidad)

(Ver A nexos).

COORDINACIÓN DEL RNP CON LA SECRETARÍA DE SALUD

A I momento no existe una coordinación sistemática entre el RN P y la Secretaría de Salud, pero existe una iniciativa para establecer convenios entre ambos organismos y para coordinar sus ac- ciones, ya que la Secretaría de Salud preten de basar su información a través del número de tarjeta de identidad de cada persona, por lo cual es indispensable esta coordinación.

Con respecto a lo instrumentos de registros utilizados por la Secretaría de Salud y el RN P, específicamente en el registro de los nacimientos en el caso de los datos de madre como ser: R esidencia y edad para ambos registros es igual ya que es el mismo instrumento (Ver A nexos, Hoja de Reporte Estadístico de N acimiento).

El Registro $\mathrm{N}$ acional de las Personas, la Secretaría de Salud y el Instituto $\mathrm{N}$ acional de Estadísticas, actualmente no tienen publicaciones con respecto a los registros vitales. 